\title{
FUM1-A Gene Required for Fumonisin Biosynthesis But Not for Maize Ear Rot and Ear Infection by Gibberella moniliformis in Field Tests
}

\author{
A. E. Desjardins, ${ }^{1}$ G. P. Munkvold, ${ }^{2}$ R. D. Plattner, ${ }^{1}$ and R. H. Proctor ${ }^{1}$ \\ ${ }^{1}$ Mycotoxin Research, National Center for Agricultural Utilization Research, United States Department of Agriculture- \\ Agricultural Research Service (USDA-ARS), Peoria, IL 61604 U.S.A.; ${ }^{2}$ Department of Plant Pathology, lowa State \\ University, Ames 50010 U.S.A.
}

Submitted 28 March 2002. Accepted 11 July 2002.

\begin{abstract}
We have analyzed the role of fumonisins in infection of maize (Zea mays) by Gibberella moniliformis (anamorph Fusarium verticillioides) in field tests in Illinois and Iowa, United States. Fumonisin-nonproducing mutants were obtained by disrupting FUM1 (previously FUM5), the gene encoding a polyketide synthase required for fumonisin biosynthesis. Maize ear rot, ear infection, and fumonisin contamination were assessed by silk-channel injection in 1999 and 2000 and also by spray application onto maize silks, injection into maize stalks, and application with maize seeds at planting in 1999. Ear rot was evaluated by visual assessment of whole ears and by calculating percentage of symptomatic kernels by weight. Fumonisin levels in kernels were determined by high-performance liquid chromatography. The presence of applied strains in kernels was determined by analysis of recovered isolates for genetic markers and fumonisin production. Two independent fumonisin-nonproducing (fum 1-3 and fum 1-4) mutants were similar to their respective fumonisin-producing (FUM1-1) progenitor strains in ability to cause ear rot following silkchannel injection and also were similar in ability to infect maize ears following application by all four methods tested. This evidence confirms that fumonisins are not required for $G$. moniliformis to cause maize ear rot and ear infection.
\end{abstract}

Additional keyword: gene disruption.

Gibberella moniliformis (synonym G. fujikuroi mating population A, anamorph Fusarium verticillioides synonym $F$. moniliforme) is an important pathogen of maize (Zea mays) worldwide (Munkvold and Desjardins 1997). G. moniliformis not only can cause severe ear rot but also can infect maize ears, stalks, and roots without causing obvious symptoms. As an ear rot pathogen, G. moniliformis reduces yield and quality

Corresponding author: A. E. Desjardins, Telephone: 309-681-6378; Fax: 309-681-6689; E-mail: desjarae@ ncaur.usda.gov.

Disclaimer: Names are necessary to report factually on available data; however, the USDA neither guarantees nor warrants the standard of the products, and the use of the name by USDA implies no approval of the product to the exclusion of others that may also be suitable.

This article is in the public domain and not copyrightable. It may be freely reprinted with customary crediting of the source. The American Phytopathological Society, 2002. of infected grain and produces mycotoxins such as fumonisins in grain. Fumonisins are a family of polyketide mycotoxins produced by $G$. moniliformis and by certain other species of the genus Fusarium, including $F$. anthophilum, $F$. fujikuroi, $F$. nygamai, $F$. oxysporum, and $F$. proliferatum (Munkvold and Desjardins 1997). Most naturally occurring strains of $G$. moniliformis produce high levels of fumonisin $\mathrm{B}_{1}\left(\mathrm{FB}_{1}\right)$ and lower levels of other fumonisin homologues, including fumonisin $\mathrm{B}_{2}\left(\mathrm{FB}_{2}\right)$, which has no oxygen atom at carbon 10 , and fumonisin $\mathrm{B}_{3}\left(\mathrm{FB}_{3}\right)$, which has no oxygen atom at carbon 5. However, rare natural variants exist that produce no $\mathrm{FB}_{1}$ but instead produce $\mathrm{FB}_{2}$ or $\mathrm{FB}_{3}$ as the major fumonisin homologue (Desjardins et al. 1996). These $\mathrm{FB}_{2^{-}}$and $\mathrm{FB}_{3}$-producing natural variants have provided chemical markers that have been used to facilitate strain tracking during tests of maize ear rot and ear infection (Desjardins and Plattner 2000).

Fumonisins are structurally similar to AAL toxin, an important pathogenicity factor of Alternaria alternata, and to sphinganine, a sphingolipid biosynthetic intermediate (Gilchrist 1998). Fumonisins, AAL toxin, and other sphinganine-analogue mycotoxins disrupt sphingolipid metabolism in plants and in animals by inhibiting the enzyme sphinganine N-acyltransferase and causing the accumulation of sphingoid bases, which is thought to be responsible for the majority of fumonisin-induced mycotoxicoses. Consumption of fumonisins has been shown to cause leucoencephalomalacia in horses, pulmonary edema in swine, and experimental liver and kidney cancer in rodents, and has been epidemiologically associated with esophageal cancer in humans (Marasas 2001).

Although fumonisins are toxic to plants as well as animals (Lamprecht et al. 1994), their role in plant pathogenesis and other aspects of Fusarium spp. ecology is unclear. The overwhelming majority of naturally occurring strains of G. moniliformis produce fumonisins, but rare natural variants of the fungus that are unable to produce fumonisins in culture and in maize kernels have been isolated from maize from Nepal (Desjardins and Plattner 2000; Nelson et al. 1991). Genetic analysis of a fumonisin-nonproducing natural variant of $G$. moniliformis showed an association between the production of fumonisins and high levels of virulence on maize seedlings (Desjardins et al. 1995). On the other hand, fumonisin-nonproducing natural variants were able to cause maize stalk rot under greenhouse conditions (Jardine and Leslie 1999) and maize ear rot and ear infection in a small-scale field test conducted in 1997 (Desjardins and Plattner 2000). These results provided evidence that production of fumonisins is not required for G. moniliformis to infect maize and to cause ear and 
stalk rot. Some caution is needed in the interpretation of these experiments with natural variants, however, because genetically unrelated natural variants undoubtedly differ in many traits, other than fumonisin production, that affect virulence.

A more rigorous test of the importance of fumonisins in ear rot and ear infection would use strains that are identical except for a gene that confers fumonisin production. To this end, we generated isogenic fumonisin-nonproducing mutants from two field strains of $G$. moniliformis: a representative $\mathrm{FB}_{1^{-}}, \mathrm{FB}_{2^{-}}$, and $\mathrm{FB}_{3}$-producing field strain and a rare natural variant that produces $\mathrm{FB}_{2}$ instead of $\mathrm{FB}_{1}$. To generate the mutants, we disrupted FUM1 (previously designated FUM5) (Proctor et al. 1999), the gene encoding a polyketide synthase that is required for fumonisin biosynthesis. In 1999 and 2000, we conducted field tests to compare two fumonisin-producing strains and the two fumonisin-nonproducing mutants derived from them for their ability to cause maize ear rot and ear infection using four different inoculation methods.

\section{RESULTS}

\section{fum1-3 and fum1-4 mutants cause maize ear rot} and ear infection following silk-channel injection.

For the field tests in this study, we used a previously described fumonisin-nonproducing mutant, GfA2364, which has the mutant fum 1-3 allele. Mutant GfA2364 was derived from strain M-3125, which has the wild-type FUM1-1 allele and produces $\mathrm{FB}_{1}, \mathrm{FB}_{2}$, and $\mathrm{FB}_{3}$. We also used a new fumonisin-nonproducing mutant GfA2556, which has a second mutant allele fum1-4. Mutant GfA2556 was derived from strain 109-R-7, which has a functional FUM1-1 allele and produces only $\mathrm{FB}_{2}$ (Table 1). We compared the ability of these FUM1-1 strains and the fum 1-3 and fum 1-4 mutants derived from them to cause maize ear rot, fumonisin contamination, and ear infection following silk-channel injection in Illinois in 1999 (field test 1) and 2000 (field test 3) and in Iowa in 1999 (field test 2).

Mean values of ear rot following silk-channel injection for the three field tests are presented in Table 2. Ear rot, as measured by visual estimation, was low in field tests 1 and 2 , and there were no significant differences $(P=0.05)$ among treatment groups (data not shown). Ear rot, as measured by percent symptomatic kernels by weight, also was relatively low in field tests 1 and 2, but was increased significantly above controls by some treatments. Injection of strain M-3125 and its mutant GfA2364 in field test 1 caused significantly higher levels of ear rot than controls when measured by percent symptomatic kernels by weight, but the fungal-inoculated treatments were not significantly different from each other. Injection of strain M-3125, mutant GfA2364, or an equal mixture of both strains in field test 3 caused significantly higher levels of ear rot than controls by visual estimation and by percent symptomatic kernels by weight, but the fungal-inoculated treatments were not significantly different from each other.

Mean fumonisin levels for the three field tests also are presented in Table 2. Mean fumonisin levels following silk-channel injection of FUM1-1 strains ranged up to fumonisins at a mean of $236 \mu \mathrm{g} / \mathrm{g}$ and $27 \%$ ear rot by weight (Table 2). Over the three field tests, kernels from control ears and from ears injected with strain M-3125 contained approximately $70 \% \mathrm{FB}_{1}$, with lower percentages of $\mathrm{FB}_{2}$ and $\mathrm{FB}_{3}$. As expected, kernels from ears injected in field tests 1 and 2 with the $\mathrm{FB}_{2}$-producing strain 109-R7 contained a mean of 94 to $99 \% \mathrm{FB}_{2}$. Mean fumonisin levels following the application of mutants GfA2364 and GfA2556 were $\leq 1 \mu \mathrm{g} / \mathrm{g}$ over all field tests, and also were less than or equal to the fumonisin levels of the control ears in each individual field test. Injection of an equal mixture of strain M-3125 and its mutant GfA2364 in field test 3 produced kernels that contained fumonisins at a mean of $104 \mu \mathrm{g} / \mathrm{g}$, which was significantly less $(P=0.05)$ than $236 \mu \mathrm{g} / \mathrm{g}$ for strain $\mathrm{M}-3125$ alone.

We compared the ability of the two FUM1-1 strains and the fum 1-3 and fum 1-4 mutants derived from them to cause maize ear infection by characterizing $357 \mathrm{G}$. moniliformis isolates recovered from maize kernels following silk-channel injection (Fig. 1). Due to the ubiquitous infection of nonsymptomatic maize kernels, G. moniliformis was isolated at high frequencies from both symptomatic and nonsymptomatic kernels from all treatment and control plots (data not shown). The frequency of FUM1-1 isolates among recovered strains was determined by analysis for genetic markers specific for this allele. The genetic markers used, however, do not distinguish between FUM1 in applied strains M-3125 and 109-R-7 and the large endemic population of $\mathrm{FB}_{1}$-producing isolates of $G$. moniliformis. Thus, the frequency of the $\mathrm{FB}_{2}$-producing strain 109R-7 among recovered isolates was determined in field test 1 by analyzing fumonisin production of a total 73 isolates recovered from plots inoculated with strain $109-\mathrm{R}-7$ or its mutant GfA2556. $\mathrm{FB}_{2}$-producing isolates comprised $82 \%$ of isolates recovered from kernels following silk-channel injection of strain 109-R-7, but none of the isolates recovered from kernels following injection of mutant GfA2556. These data indicate that the fum 1-4 allele, although produced by a single recombination event, was stable under field conditions.

The frequency of fumonisin-nonproducing mutants among recovered isolates was determined by analysis for genetic markers specific for fum 1-3 and fum 1-4 alleles. Of the isolates recovered after injection of mutant GfA2364 in the three field tests, 85 to $100 \%$ carried the fuml-3 allele. Injection of an equal mixture of strain M-3125 and mutant GfA2364 in field test 3 produced $35 \%$ fum $1-3$ mutants among recovered isolates. Of the isolates recovered after injection of mutant GfA2556 in field test 1, 74\% carried the fum 1-4 allele, which was not significantly different $(P=0.05)$ from $82 \%$ recovery of the $\mathrm{FB}_{2}$-producing progenitor strain 109-R-7 from plots where it was applied. Of the isolates recovered after injection of mutant GfA2556 in field test 2, 93\% carried the fum1-4 allele.

fum 1-3 and fum 1-4 mutants were recovered at low frequencies throughout the plots of field tests 1 and 2, indicating that the mutants could move, during the months between inoculation and harvest, from the plots where they had been applied. fum 1-3 mutants were recovered at a frequency of $44 \%$ from control plots in field test 3. The higher recovery of mutant GfA2364 in control plots in field test 3 may reflect the considerably higher level of ear rot in 2000 than in field tests 1 and 2

Table 1. Strains of Gibberella moniliformis used in this study ${ }^{\mathrm{y}}$

\begin{tabular}{|c|c|c|c|c|}
\hline Strain & FUM1 genotype & Fumonisin phenotype & Source & Reference \\
\hline M-3125 & FUM1-1 & $\mathrm{FB}_{1}, \mathrm{FB}_{2}, \mathrm{FB}_{3}$ & Field strain from maize & Proctor et al. 1999 \\
\hline 109-R-7 & FUM1-1 & $\mathrm{FB}_{2}$ & Progeny of cross between field strains from maize & Desjardins et al. 1996 \\
\hline GfA2364 & fuml-3 & None detected ${ }^{\mathrm{z}}$ & Gene replacement in M-3125 & Proctor et al. 1999 \\
\hline GfA2556 & fum1-4 & None detected ${ }^{\mathrm{z}}$ & Gene disruption in $109-\mathrm{R}-7$ & This study \\
\hline
\end{tabular}

\footnotetext{
${ }^{y}$ The prefix M refers to the Fusarium Research Center, The Pennsylvania State University, University Park.
}

${ }^{\mathrm{z}}$ None detected $=$ at least a $99.9 \%$ reduction in fumonisin production in culture. 
in 1999. As expected, fum 1-4 mutants were absent from field test 3, because the fum1-4 mutant was not tested in 2000 .

fum 1-3 and fum 1-4 mutants are similar to wild-type FUM1-1 strains in causing maize ear infection following silk spray application, stalk injection, and seed application.

In field tests 1 and 2, we tested three additional inoculation methods. We compared the $\mathrm{FB}_{1^{-}}, \mathrm{FB}_{2^{-}}$, and $\mathrm{FB}_{3}$-producing FUM1-1 strain $\mathrm{M}-3125$, the $\mathrm{FB}_{2}$-producing FUM1-1 strain 109-R-7, the fumonisin-nonproducing fum1-3 mutant GfA2364, and the fumonisin-nonproducing fum1-4 mutant GfA2556 for ability to cause maize ear rot, fumonisin contamination, and ear infection following silk spray application, stalk injection, and application to seed at planting.

For all four strains and three application methods, mean values of ear rot were low and were not significantly different from controls (data not shown). Mean values of fumonisin levels are presented in Table 3. Application of fumonisin-producing strains by all three methods was not associated with higher levels of fumonisins than in control ears. Application of both fumonisin-nonproducing mutants by silk spray significantly reduced fumonisin levels to below $1 \mu \mathrm{g} / \mathrm{g}$ in field test 2 , but not in field test 1 . Application of fumonisin-nonproducing mutants by stalk injection or seed application was not associated with lower levels of fumonisins than in control ears.

We compared the ability of the two FUMI-1 strains and the fum 1-3 and fum 1-4 mutants derived from them to cause maize ear infection by characterizing $778 \mathrm{G}$. moniliformis isolates recovered from maize kernels following application by silk spray, stalk injection, and seed application (Fig. 2). The frequency of the FUM1-1 genotype among recovered isolates, either applied strains or endemic isolates, was determined by analysis for genetic markers specific for this allele. The frequency of kernel infection by the $\mathrm{FB}_{2}$-producing strain 109-R7 was determined in field test 1 , as described above, by analyzing fumonisin production of a total of 253 recovered isolates. $\mathrm{FB}_{2}$-producing isolates comprised 64,62 , and $34 \%$ of isolates recovered from kernels following application of strain 109-R-7 to silks, stalks, and seeds, respectively. Fumonisin $\mathrm{B}_{2-}$ producing isolates comprised a mean of $7 \%$ of isolates recovered following application of mutant GfA2556, probably indicating that strain 109-R-7 had moved from plots where it had been applied.

The frequency of kernel infection by the fumonisin-nonproducing mutants after silk spray was determined by analysis of recovered isolates for genetic markers specific for fum 1-3 and fum 1-4 alleles. Of the isolates recovered after silk spray application of mutant GfA2364 in field tests 1 and 2, 88 and 70\%, respectively, carried the fum1-3 allele. Of the isolates recovered after silk spray application of mutant GfA2556 in field test $1,45 \%$ carried the fuml-4 allele, which was not significantly different $(P=0.05)$ from $64 \%$ recovery of the $\mathrm{FB}_{2}$-pro- ducing progenitor strain 109-R-7. Of the isolates recovered after silk spray application of mutant GfA2556 in field test 2, 96\% carried the fum1-4 allele.

Stalk injection and seed application in field test 2 did not significantly increase the frequency of recovery of mutant GfA2364 or GfA2556 compared with controls. Stalk injection and seed application in field test 1 significantly increased the frequency of recovery of mutant GfA2364 to 46 and 30\%, respectively. Stalk injection in field test 1 increased the frequency of recovery of mutant GfA2556 to $40 \%$, which was not significantly different from $62 \%$ recovery of strain 109-R-7. The frequency of recovery of mutant GfA2556 after seed application was $2 \%$, which was not significantly different $(P=$ 0.05 ) from the $34 \%$ recovery of strain 109-R-7.

\section{DISCUSSION}

Mycotoxins are defined as low-molecular-weight fungal metabolites that are toxic to animals. Consumption of mycotoxins can adversely affect the health of animals and humans; therefore, mycotoxin research historically has been performed largely by natural product chemists, animal toxicologists, and human disease epidemiologists. Recently, plant pathologists have begun to investigate whether mycotoxins play a role in plant pathogenesis in addition to their role in animal diseases. Four mycotoxin classes of particular interest to both animal pathologists and plant pathologists worldwide are ergot alkaloids of the genus Neotyphodium and related fungi, aflatoxins of the genus Aspergillus, and trichothecenes and fumonisins of the genus Fusarium (Desjardins and Hohn 1997). During the past few years, biosynthetic genes for each of these four major classes have been disrupted to produce mycotoxin-nonproducing mutants. With the completion of the present study, gene disruption mutants now have been used to test the roles of three mycotoxin classes in plant pathogenesis and in other aspects of plant-fungal interactions, with dramatic results.

Ergotism, the most notorious mycotoxicosis in history, is caused by consumption of cereal grains and pasture grasses that are contaminated by ergot alkaloids. Recently, $\operatorname{lps} A$, a peptide synthetase gene required for ergovaline biosynthesis, was cloned and disrupted in Neotyphodium spp. Lp1, which systemically infects perennial rye grass (Panaccione et al. 2001). Replacement of $\operatorname{lps} A$ eliminated ergovaline production but did not affect systemic infection of perennial rye grass. Thus, this mycotoxin appears not to be essential for this aspect of the plant-fungal interaction. The opposite conclusion has been reached in similar gene disruption studies of trichothecene mycotoxins of Fusarium spp. Trichothecenes are potent inhibitors of protein synthesis and are highly toxic to animals and plants. Because of their acute phytotoxicity, trichothecenes were strong candidates as virulence factors in plant diseases caused by a wide range of Fusarium spp. Elimination of trichothecene

Table 2. Mean ear rot values and fumonisin levels in maize kernels following silk-channel injection with Gibberella moniliformis

\begin{tabular}{|c|c|c|c|c|c|c|c|}
\hline \multirow[b]{2}{*}{ Treatment $^{x}$} & \multicolumn{2}{|c|}{ Field test 1} & \multicolumn{2}{|c|}{ Field test 2} & \multicolumn{3}{|c|}{ Field test 3} \\
\hline & Ear rot $(w t \%)^{y}$ & FB $(\mu \mathrm{g} / \mathrm{g})^{\mathrm{z}}$ & Ear rot $(w t \%)$ & FB $(\mu \mathrm{g} / \mathrm{g})$ & Rating & Ear rot $(w t \%)$ & FB $(\mu \mathrm{g} / \mathrm{g})$ \\
\hline Control & $6.6 \mathrm{c}$ & $1.1 \mathrm{~cd}$ & $0.8 \mathrm{~b}$ & $2.4 \mathrm{c}$ & $2.9 \mathrm{~b}$ & $9.6 \mathrm{~b}$ & $10.1 \mathrm{c}$ \\
\hline M-3125 & $15.8 \mathrm{a}$ & $39.3 \mathrm{a}$ & $2.6 \mathrm{ab}$ & $26.2 \mathrm{a}$ & $4.4 \mathrm{a}$ & $26.8 \mathrm{a}$ & $236 \mathrm{a}$ \\
\hline GfA2364 & $15.0 \mathrm{a}$ & $0.1 \mathrm{e}$ & $3.5 \mathrm{a}$ & $0.3 \mathrm{~d}$ & $4.4 \mathrm{a}$ & $28.6 \mathrm{a}$ & $1.0 \mathrm{c}$ \\
\hline M-3125/GfA2364 & $\ldots$ & $\ldots$ & $\ldots$ & $\ldots$ & $4.6 \mathrm{a}$ & $27.2 \mathrm{a}$ & $104 \mathrm{~b}$ \\
\hline 109-R-7 & $12.3 \mathrm{ab}$ & $8.0 \mathrm{~b}$ & $0.9 \mathrm{~b}$ & $9.6 \mathrm{ab}$ & $\ldots$ & $\ldots$ & $\ldots$ \\
\hline GfA2556 & $8.1 \mathrm{c}$ & $0.5 \mathrm{de}$ & $2.1 \mathrm{ab}$ & $0.1 \mathrm{~d}$ & $\ldots$ & $\ldots$ & $\ldots$ \\
\hline
\end{tabular}

$\mathrm{x}$ The designation M-3125/GfA2364 indicates an equal mixture of the two strains M-3125 and GfA2364.

y A disease severity rating scale from 1 to 7 was based on visual estimation of infected kernels on each ear, and percent ear rot by weight was determined by dividing weight symptomatic kernels by the weight of all kernels from each ear $(\times 100)$. For each field test, numbers in each column followed by the same letter are not significantly different $(P=0.05)$.

${ }^{\mathrm{z}}$ Fumonisin levels in kernels were determined by high-performance liquid chromatography. 
production by disruption of the sesquiterpene cyclase gene TRI5 has indeed demonstrated a role for trichothecenes in enhancing virulence of $F$. sporotrichioides and Gibberella pulicaris (anamorph $F$. sambucinum) on parsnip root, and of $G$. zeae (anamorph $F$. graminearum) on wheat and maize ears (Desjardins and Hohn 1997; Harris et al. 1999). On the other hand, trichothecenes are not essential for virulence of $G$. pulicaris on potato tubers (Desjardins and Hohn 1997). An important role for fumonisins in virulence of $G$. moniliformis was supported by their phytotoxicity and, in particular, by their similarity in structure and activity to the sphinganine analogue, AAL toxin of A. alternata f. sp. lycopersici, which is a pathogenicity factor on tomato (Gilchrist 1998). In this study, we have confirmed through gene disruption that fumonisins are not essential for maize ear rot and ear infection by $G$. moniliformis under field conditions. These experiments also have confirmed the requirement for the polyketide synthase encoded by FUM1 for fumonisin biosynthesis in maize ears under field conditions.

Biosynthesis of fumonisins from acetate and alanine involves a complex series of oxygenations, esterifications, amino-transfer, and other reactions. In common with other mycotoxin pathway genes, at least five fumonisin pathway genes appear to be localized in a gene cluster (Seo et al. 2001). Our current hypothesis is that the polyketide synthase encoded by FUM1 catalyzes the synthesis of the fumonisin backbone from carbon 3 to carbon 20, which precursor-feeding experiments indicate is derived from acetate (Blackwell et al. 1994). In earlier articles (Proctor et al. 1999; Seo et al. 2001), this polyketide synthase gene was designated FUM5. However, we recently determined by mutant complementation analysis that FUM5 is the same gene as Fuml, a locus that was previously defined by meiotic analysis of a fumonisin-nonproducing fum1-2 natural variant (Desjardins et al. 1996; Proctor et al. 1999; and R. Proctor, R. Plattner, and A. Desjardins, unpublished data). Therefore, FUM5 has been renamed FUM1. FUM1 disruptants and the fum1-2 natural variant are 99.9 to $100 \%$ reduced in fumonisin production in culture. A small-scale field test (40 maize ears) in 1997 indicated that the fumonisin-nonproducing natural variant was able to cause ear rot following silk-channel injection, and did not produce any fumonisins in maize kernels (Desjardins and Plattner 2000). This preliminary test provided the first evidence that production of fumonisins was not required for maize ear rot by $G$. moniliformis.

Results from the 1997 field test, however, were not conclusive because the field test of the fumonisin-nonproducing

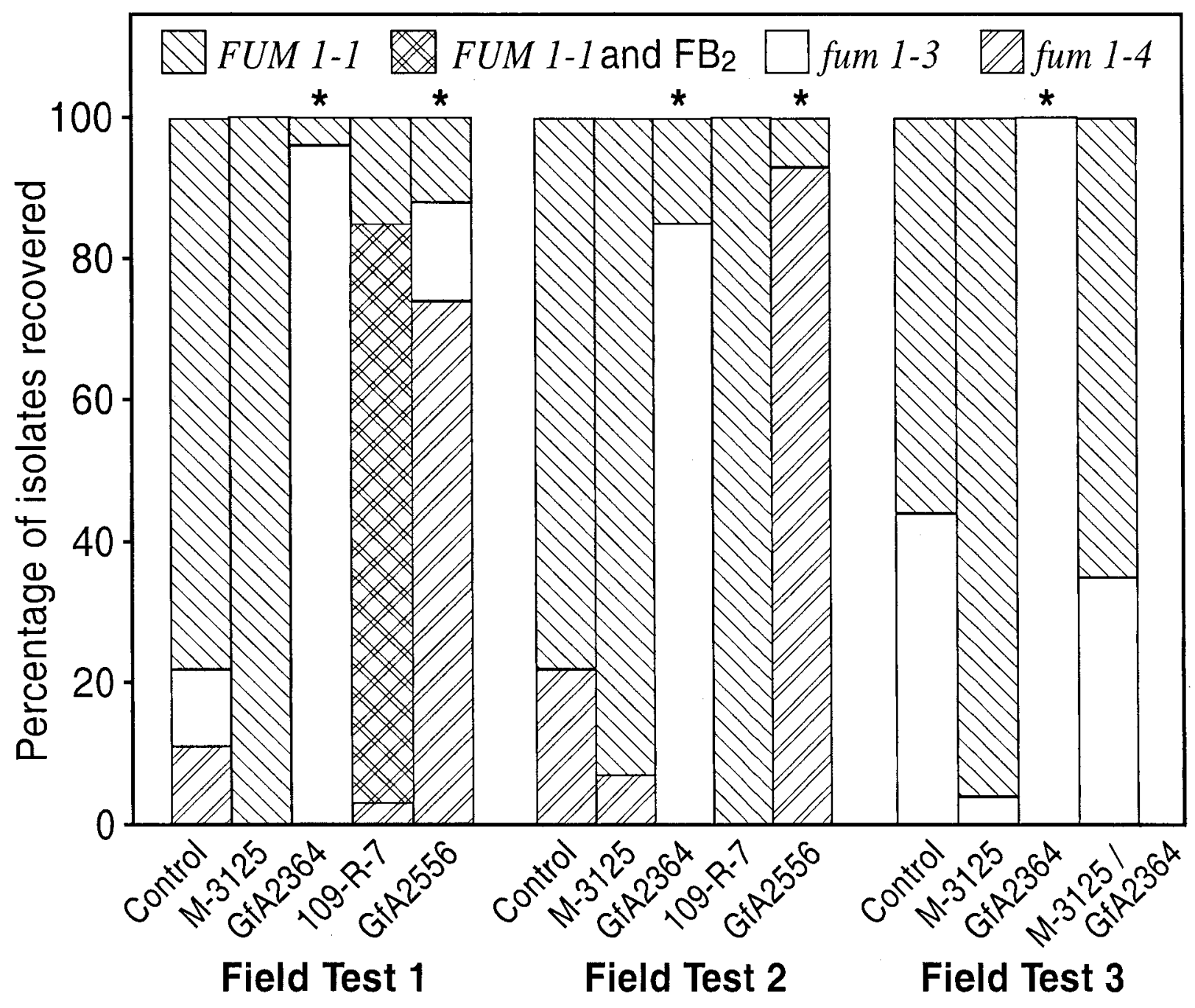

Fig. 1. Mean frequency of isolates of Gibberella moniliformis among isolates recovered from maize kernels following silk-channel injection. Isolates were analyzed for FUM1 genotype using genetic markers specific for each allele, and for fumonisin phenotype by high-performance liquid chromatography of culture extracts. For each field test, bars topped with an asterisk indicate that recovery of the indicated fuml-3 or fum1-4 mutant was significantly different from control $(P=0.05)$. The numbers of isolates tested for each treatment are (from left to right for each field test) field test 1: 18, 23, 23, 38, and 35; field test 2: $13,23,25,25$, and 29; and field test 3: 25, 25, 29, and 26 . 
natural variant was conducted at only one site, the number of maize plants tested was small, and the analysis of strains recovered from maize kernels was incomplete. Furthermore, the fumonisin-producing and nonproducing strains tested were natural variants and, thus, not isogenic. All of these limitations were overcome in the present study. Cloning of FUM1 in 1999 provided the essential tool to create isogenic lines that differ only in fumonisin production, in two different genotypes of $G$. moniliformis. Three larger-scale field tests then were designed at sites in Illinois and Iowa to test isogenic lines in both genetic backgrounds and to include four different inoculation methods. Finally, a complete microbiological analysis was conducted for each field test.

The present study establishes that fumonisin-nonproducing strains of $G$. moniliformis are able to cause maize ear rot and ear infection. All strains tested caused low to moderate levels of ear rot following application by silk-channel injection, and there were no significant differences in ear rot rating between each of the fumonisin-producing strains and the fumonisinnonproducing mutant derived from it, or an equal mixture of one strain with its mutant. Our data confirm previous reports that both silk-channel injection and silk-spray application are effective methods for maize kernel infection with $G$. moniliformis (Munkvold et al. 1997). Our data also provide further evidence that a nearly complete replacement of endemic isolates is necessary for a fumonisin-nonproducing strain to significantly decrease fumonisin levels (Desjardins and Plattner 2000). For example, silk-channel injection replaced an average of $90 \%$ of endemic isolates with fumonisin-nonproducing mutants, and reduced fumonisin levels in the kernels in all five cases. Silk spray application replaced an average of $75 \%$ of endemic isolates with fumonisin-nonproducing mutants, and fumonisin levels in the kernels were reduced, but the reductions were significant in only two of four cases. Although stalk injection and seed application at planting did, in some treatments, increase recovery of fumonisin-nonproducing mutants, fumonisin levels in the kernels were not significantly reduced. For each of the four application methods, frequencies of the $\mathrm{FB}_{2}$-producing strain 109-R-7 and the fumonisin-nonproducing mutant GfA2556 among recovered strains were not significantly different. However, the microbiological analysis used in the present study provides only an estimate of the extent of infection of maize kernels; thus, we cannot conclude from our data that fumonisins have no effect on the infection of maize by G. moniliformis.

Fumonisin-producing strains of G. moniliformis are closely associated with maize at all developmental stages. This fungus is not only the most common pathogen of maize; it also is among the most common fungi found colonizing symptomless maize plants (Munkvold and Desjardins 1997). Although the relationship between $G$. moniliformis and maize is not as intimate as that between Neotyphodium spp. and perennial rye grass, there are similarities. In particular, in both systems, the mycotoxin that plays such an important role in animal toxico- sis is not required for plant infection and pathogenesis. Infection with Neotyphodium spp. is beneficial to plant hosts, enhancing fitness and resistance to pathogens and herbivores (Schardl 1996). Any contributions of $G$. moniliformis infection, in general, or of fumonisin production, in particular, to fitness and disease resistance of maize plants remain to be demonstrated. Fumonisin-nonproducing mutants provide essential tools to investigate the role of fumonisins in all aspects of the interaction of $G$. moniliformis with maize.

\section{MATERIALS AND METHODS}

\section{Fungal strains and inoculum preparation.}

The G. moniliformis strains used in this study are described in Table 1. The virulent wild-type strain M-3125 was selected as a representative $\mathrm{FB}_{1^{-}}, \mathrm{FB}_{2^{-}}$, and $\mathrm{FB}_{3}$-producing strain. Strain M-3125 was isolated from maize in California and was the progenitor of the previously described fumonisin-nonproducing mutant GfA2364 (Proctor et al. 1999). Mutant GfA2364 was generated by replacing the wild-type FUM1-1 allele in strain M-3125 with the mutant allele fum 1-3. This was accomplished via transformation-mediated gene disruption that involved a double homologous recombination event between the FUM1-1 allele and disruption vector pB6CS-Hyg. The fum 1-3 allele differed from the wild-type allele in that a 530-bp HindIII fragment near the middle of the coding region was replaced with the hygromycin resistance gene, $H y g B$. Strain GfA2364 was more than $99.9 \%$ reduced in fumonisin production on cracked maize and in liquid culture (Proctor et al. 1999).

The virulent strain $109-\mathrm{R}-7$ was an $\mathrm{FB}_{2}$-producing strain. Strains that produce primarily $\mathrm{FB}_{2}$, with no $\mathrm{FB}_{1}$ or $\mathrm{FB}_{3}$, are rare in the endemic population of G. moniliformis in the U.S. midwestern states (Desjardins and Plattner 2000). Thus, production of high levels of $\mathrm{FB}_{2}$, with little or no $\mathrm{FB}_{1}$ or $\mathrm{FB}_{3}$, can be used as a marker to facilitate tracking of strain 109-R-7. Strain 109-R-7 was derived as an ascospore from a cross between strains from maize from California and South Carolina (Desjardins et al. 1996) and was the progenitor of the fumonisin-nonproducing mutant GfA2556. The FUM1 gene of strain 109-R-7 has not been sequenced and may differ at the nucleotide level from the FUM1-1 allele of strain M-3125. Both genes, however, confer wild-type levels of fumonisins; thus, both will be designated as wild-type FUM1-1 alleles in this study. In mutant GfA2556, the functional FUM1-1 allele was replaced with the mutant fum 1-4 allele. The fum 1-4 allele was generated via transformation-mediated gene disruption, but resulted from a single homologous recombinational event between the functional FUM1-1 allele in 109-R-7 and the disruption vector pB6CS-Hyg. The mutant fum1-4 allele consisted of two truncated copies of the FUM1 coding region separated from one another by bacterial vector (pBluescriptII) sequences. One truncated copy in fuml-4 lacked the first 249 nt of the coding region and the other lacked the last 3,156 nt.

Table 3. Mean fumonisin levels $(\mu \mathrm{g} / \mathrm{g})$ in maize kernels following silk-spray application, stalk application, and seed application with Gibberella moniliformis

\begin{tabular}{|c|c|c|c|c|c|c|}
\hline \multirow[b]{2}{*}{ Treatment $^{\mathrm{z}}$} & \multicolumn{3}{|c|}{ Field test 1} & \multicolumn{3}{|c|}{ Field test 2} \\
\hline & Silk & Stalk & Seed & Silk & Stalk & Seed \\
\hline Control & $3.6 \mathrm{abc}$ & $3.7 \mathrm{abc}$ & $1.1 \mathrm{~cd}$ & $6.2 \mathrm{bcd}$ & $3.1 \mathrm{~cd}$ & $1.9 \mathrm{~d}$ \\
\hline M-3125 & $5.6 \mathrm{a}$ & $4.5 \mathrm{abc}$ & $3.4 \mathrm{abc}$ & $12.6 \mathrm{ab}$ & $6.0 \mathrm{bcd}$ & $1.7 \mathrm{~d}$ \\
\hline GfA2364 & $1.2 \mathrm{~cd}$ & $1.6 \mathrm{~cd}$ & $1.2 \mathrm{~cd}$ & $0.7 \mathrm{e}$ & $1.7 \mathrm{~d}$ & $5.8 \mathrm{bcd}$ \\
\hline 109-R-7 & $4.1 \mathrm{abc}$ & $2.0 \mathrm{abc}$ & $2.0 \mathrm{bcd}$ & $4.1 \mathrm{bcd}$ & $2.1 \mathrm{~d}$ & $8.3 \mathrm{abc}$ \\
\hline GfA2556 & $1.8 \mathrm{bcd}$ & $1.2 \mathrm{~cd}$ & $0.8 \mathrm{~cd}$ & $0.3 \mathrm{e}$ & $2.5 \mathrm{~cd}$ & $5.8 \mathrm{bcd}$ \\
\hline
\end{tabular}

${ }^{\mathrm{z}}$ Fumonisin levels in kernels were determined by high-performance liquid chromatography. For each inoculation method in each field test, numbers in each column followed by the same letter are not significantly different $(P=0.05)$. 


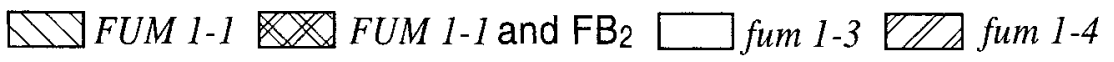

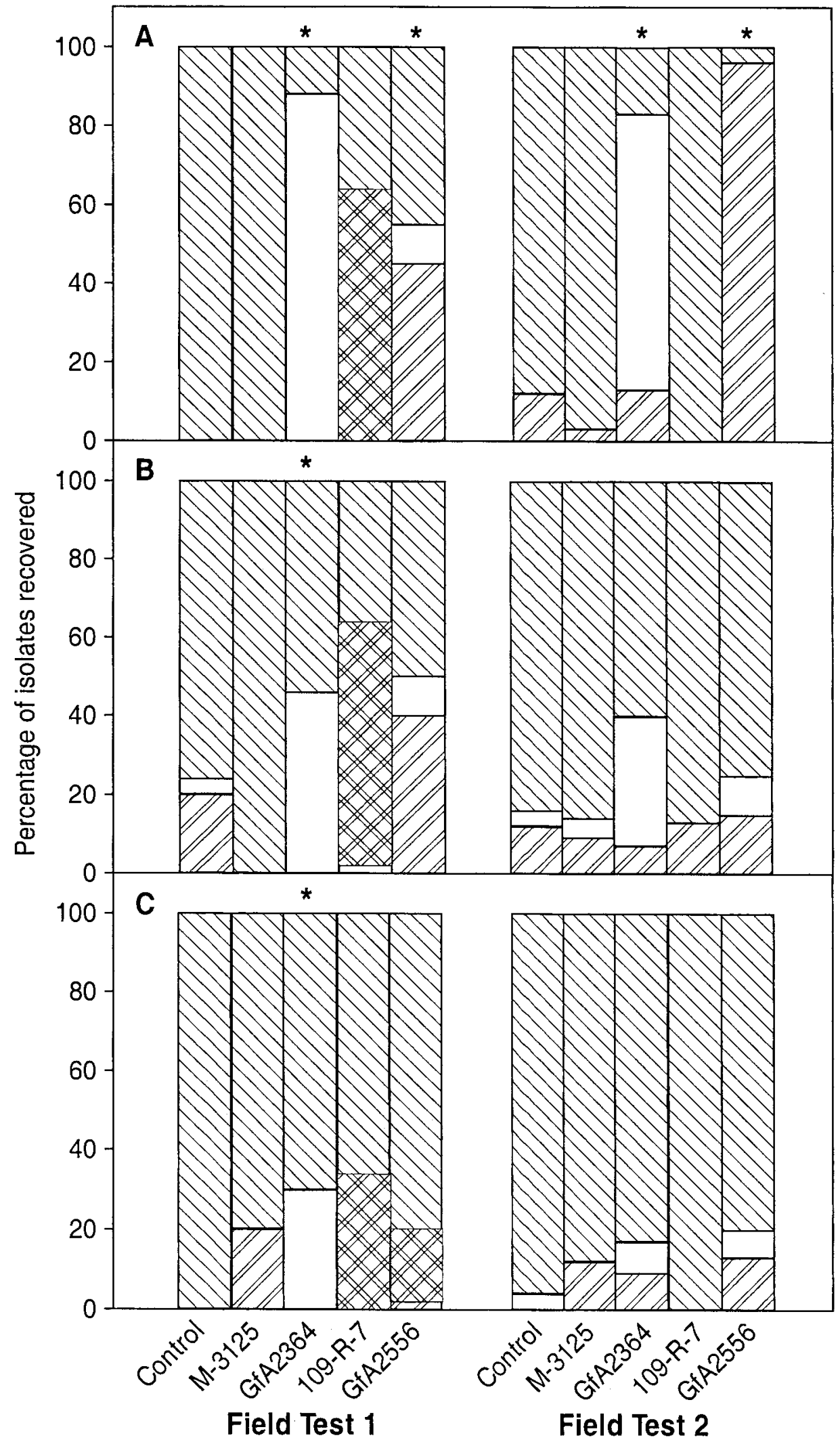

Fig. 2. Mean frequency of isolates of Gibberella moniliformis among isolates recovered from maize kernels following A, silk-spray application, B, stalk injection, and $\mathbf{C}$, seed application at planting. Isolates were analyzed for FUM1 genotype using genetic markers specific for each allele, and for fumonisin phenotype by high-performance liquid chromatography of culture extracts. For each application method and each field test, bars topped with an asterisk indicate that recovery of the indicated fuml-3 or fum 1-4 mutant was significantly different from control $(P=0.05)$. The numbers of isolates tested for each treatment are (from left to right for each inoculation method) silk spray application: 21, 22, 25, 42, 38, 24, 24, 23, 25, and 22; stalk injection: 20, 23, 24, 42, 42, 24, 24, 21, 20, and 22; and seed application: 15, 20, 23, 41, 50, 20, 19, 18, 22, and 22. 
Also, in the copy with the $5^{\prime}$ truncation, the 530-bp HindIII fragment near the middle of the coding region was replaced with $\mathrm{HygB}$ (Proctor et al. 1999). Extensive efforts to obtain the more stable fum 1-3 mutant allele in the strain 109-R-7 genetic background were unsuccessful. However, the fum 1-4 allele in mutant GfA2556 appeared to be stable during experiments in the laboratory and under field test conditions. Mutant GfA2556 was more than $99.9 \%$ reduced in fumonisin production when grown for 3 weeks on cracked maize under standard conditions.

Spore suspensions for silk-channel injections, silk spray, and stalk injections were produced by washing spores (macroconidia and microconidia) from cultures grown on V8 juice agar for 7 to 10 days. Spore suspensions were diluted for field application in two different ways. For field tests 1 and 2, spore suspensions were diluted with water to a final concentration of $1 \times 10^{5} \mathrm{spores} / \mathrm{ml}$. For field test 3, spore suspensions were prepared in modified Bilay's medium (Harris et al. 1999) to a final concentration of $5 \times 10^{6}$ spores $/ \mathrm{ml}$ for each strain applied. Spore suspensions were prepared in the laboratory and applied in the field on the same day. For application with seeds in the 1999 field test, fungal-infested toothpicks were prepared in Bilay's medium as previously described (Desjardins and Plattner 2000).

\section{Field tests.}

Three field tests were conducted: test 1 in Peoria County, IL in 1999; test 2 in Boone County, IA in 1999; and test 3 in Peoria County, IL in 2000. Field tests were conducted under terms outlined in permit 98-355-01R and permit 00-004-01R granted by United States Department of Agriculture, Animal and Plant Health Inspection Service, Biotechnology Program. Commercial yellow maize hybrid 3394 (Pioneer Hibred International, Inc.) was used for all three field tests. Field test size was limited and blocks were arranged to minimize the potential for environmental release of genetically engineered fungal strains.

Field tests 1 and 2 were replicates, and compared the ability of fungal strains M-3125, 109-R-7, GfA2364, and GfA2556 to cause ear rot and ear infection using four different inoculation methods. All spore suspensions were $1 \times 10^{5} \mathrm{spores} / \mathrm{ml}$ in water. For application to the silk channel, $2 \mathrm{ml}$ of spore suspension were injected into the center of the silk channel inside the cavity and above the cob of the primary ear 4 to 6 days after silk emergence (Reid et al. 1996). Control ears were injected with water. For application to silks, 2 to $3 \mathrm{ml}$ of spore suspension were sprayed onto silks at 4 to 6 days after silk emergence, and again 3 days later. Control ears were sprayed with water. After spraying, ears were loosely covered with plastic bags for 2 days. For application to stalks, $2 \mathrm{ml}$ of spore suspension was injected into the second internode above the soil line at growth stage V8 (Ritchie et al. 1997). Control stalks were injected with water. For application with seed, a fungalinfested toothpick was buried completely in the soil adjacent to each seed at planting. Control seed were planted next to a toothpick soaked in sterile Bilay's medium.

Field tests 1 and 2 were a split-plot design with the four inoculation treatments as main plots and fungal strains as subplots, with five replicates of each main plot. Each subplot was $4 \mathrm{~m}$ long, and rows were approximately $1 \mathrm{~m}$ wide. Each subplot contained 11 rows containing 15 maize plants. The six odd-numbered rows were not inoculated, and ears or stalks of the middle 10 plants of the even-numbered rows were inoculated. For seed-inoculated plots, all 15 seeds that were planted were inoculated, but only 10 plants were harvested.

Field test 3 compared the ability of fungal strains M-3125 and GfA2364, separately and in combination, to cause ear rot and ear infection following injection into the silk channel as described above. For inoculation of strain M-3125 and mutant GfA2364 separately, $2 \mathrm{ml}$ of spore suspension at $5 \times 10^{6}$ spores/ml in Bilay's medium was applied to each ear. For coinoculation, $2 \mathrm{ml}$ of spore suspension containing each strain at $5 \times 10^{6} \mathrm{spores} / \mathrm{ml}$, for a total of $10 \times 10^{6} \mathrm{spores} / \mathrm{ml}$, was applied to each ear. Control ears were injected with Bilay's medium. Field test 3 consisted of a plot of maize, with five randomly assigned replicate subplots of each of the two fungal strains and the combination being tested, and five control subplots. Plot dimensions and design were as described above.

\section{Disease ratings.}

At physiological maturity, ears from the treated plants were hand picked, husked, and air dried in the laboratory for at least 1 month. Each ear was individually evaluated using a disease severity rating scale based on visual estimation of ear rot as the percentage of visibly damaged kernels on a ear as follows: $1=0 \%, 2=1$ to $3 \%, 3=4$ to $10 \%, 4=11$ to $25 \%, 5=26$ to $50 \%, 6=51$ to $75 \%$, and $7=76$ to $100 \%$ (Reid et al. 1996). For microbiological analysis, each ear was sampled by removing 6 to 10 representative kernels, including nonsymptomatic and symptomatic kernels if both were present. Kernels from the 10 ears of each replicate plot were pooled. The remaining kernels were hand shelled and separated into a nonsymptomatic fraction and a symptomatic fraction (designated an ear rot fraction) that contained all kernels that were visibly moldy, darkened, streaked, or chalky in appearance. The nonsymptomatic fraction and the ear rot fraction of each ear were weighed separately.

\section{Fumonisin analysis.}

For fumonisin analysis, nonsymptomatic fractions and ear rot fractions from the 10 ears of each replicate plot were pooled, weighed, ground, and analyzed by high-performance liquid chromatography (HPLC) of their orthophthalaldehyde derivatives as described (Sydenham et al. 1992). The detection limits for $\mathrm{FB}_{1}, \mathrm{FB}_{2}$, and $\mathrm{FB}_{3}$ were $0.05,0.1$, and $0.1 \mu \mathrm{g} / \mathrm{g}$, respectively, of the maize dry weight. To ensure reliability and reproducibility of the analytical procedures, a reference maize meal containing fumonisin at approximately $1 \mu \mathrm{g} / \mathrm{g}$ was run as a control with every set of samples. In addition, replicate extractions and HPLC analyses were done on randomly chosen samples. Fumonisin levels in samples were reproducible across the quantitation range, with a coefficient of variation of $<20 \%$. Unless otherwise indicated, fumonisin levels are the sum of levels of $\mathrm{FB}_{1}, \mathrm{FB}_{2}$, and $\mathrm{FB}_{3}$ expressed as total micrograms per gram. Fumonisin $\mathrm{B}_{4}$, which has no oxygen atom at carbon 5 or 10 , was not quantitated in this study but was present at low levels in samples that contained $\mathrm{FB}_{1}, \mathrm{FB}_{2}$, or $\mathrm{FB}_{3}$.

The production of $\mathrm{FB}_{1}, \mathrm{FB}_{2}$, and $\mathrm{FB}_{3}$ by fungal strains was assessed in a cracked maize medium consisting of $10 \mathrm{~g}$ of maize and $4 \mathrm{ml}$ of water autoclaved in a 50-ml Erlenmeyer flask. Cultures were incubated in the dark at $25^{\circ} \mathrm{C}$ for 3 weeks, weighed, extracted with acetonitrile:water 1:1 (vol/vol), filtered, and analyzed by HPLC as described above.

\section{Identification of fungal isolates recovered from maize kernels.}

For this study, fungal isolates are defined as individual strains recovered from maize kernels and reisolated from single conidia. At least 10 kernels from the pooled sample of each replicate plot were surface disinfested with $0.5 \%$ sodium hypochlorite, split in half, and cultured on a Fusarium-selective medium (Nelson et al. 1983). One fungal isolate per kernel was reisolated from a single spore and identified as a putative G. moniliformis by production of micro- 
conidia in chains. This trait is not sufficient to distinguish $G$. moniliformis from the closely related species $F$. proliferatum and $F$. thapsinum, which also can be found in maize in the midwestern U.S. region (Munkvold and Desjardins 1997). However, for the objectives of this study, it was not necessary to identify all isolates to the species level because genetic markers and the fumonisin production profile were used to determine whether isolates were similar to the applied strains. Genomic DNA was isolated from putative $G$. moniliformis isolates as previously described (Desjardins et al. 1996). Polymerase chain reaction (PCR) employed the isolated DNA as template and primer combinations capable of specifically identifying FUM1-1, fum1-3, and fum1-4 alleles. We employed a three-primer combination that consisted of oligonucleotides rp216 (5'-GTGATGGAGGCATGGCTGTTATTGG-3') and rp217 (5'-TCCAATCCTTGCTGACTCGGCTCTC-3'), which are complementary to regions within FUM1, and oligonucleotides 1098 (5'-ACCAAGCCTATGCCTACAGCATCC-3'), which is complementary to the $3^{\prime}$ end of $H y g B$. This three-primer combination amplifies a single 780-bp DNA fragment from the wild-type FUM1-1 allele in fumonisin-producing strains M-3125 and 109-R-7, a single 580-bp DNA fragment from the fum 1-3 replacement allele in mutant GfA2364, and both DNA fragments from the fum 1-4 disrupted allele in mutant GfA2556. The PCR product band pattern from each field isolate was compared to those from wild-type and mutant strains to determine the genotype of each field isolate. All isolates that yielded the same PCR product band as strains M-3125 and 109-R-7 were considered to have the FUM1-1 allele. All isolates that yielded the same PCR band as mutant GfA2364 were considered to have the fum 1-3 allele. All isolates that yielded the same two bands as mutant GfA2556 were considered to have the fum 1-4 allele.

\section{Statistical analysis.}

For dependent variables of ear rot rating and fumonisin level, data were analyzed using Levene's homogeneity of variance test on different inoculation materials for each inoculation method, separately, and for each field test, separately. Transformations were performed to stabilize variances when appropriate. One-way analysis of variance (ANOVA) tests were performed to compare different inoculation materials for each inoculation method, separately, and for each field test, separately, for the dependent variables of ear rot rating and fumonisin level. Duncan's multiple range test was the multiple comparison procedure for determining factor level differences when preceded by a significant $F$ test result, with $\alpha$ at 0.05 .

For dependent variables of FUM1 genotype and fumonisin phenotype, data were analyzed using Levene's homogeneity of variance test on different inoculation materials for each inoculation method, separately, and for each field test, separately. All analyses were conducted on transformed data when appropriate to satisfy treatment homogeneity of variance assumptions for ANOVA $F$ tests. For ease of interpretation, all tabular values are presented as raw data even though statistical comparisons may have been performed using transformed data values. One-way ANOVA tests were performed to compare different inoculation materials for each inoculation method, separately, and for each field test, separately, for the dependent variables of FUM1 genotype and fumonisin phenotype. In cases where all five replicates were zero, they were removed from the analysis because they were automatically considered to be statistically different from any other treatment combination. Duncan's multiple range test was the multiple comparison procedure for determining factor level differences when preceded by a significant $F$ test result, with $\alpha$ at 0.05 .

\section{ACKNOWLEDGMENTS}

We thank D. Palmquist for statistical analysis and T. Harrington for technical advice; A. Ackerman, C. Biggerstaff, W. Chen, S. Folmar, C. Gravert, M. Moore, S. Poling, M. Romero, D. Shane, J. Shriver, and T. Wilson for technical assistance; the W. Christ family for assistance in conducting the Illinois field tests; and B. Brueland and D. Starrett for assistance in conducting the Iowa field test.

\section{LITERATURE CITED}

Blackwell, B., Miller, J., and Savard, M. 1994. Production of carbon 14labeled fumonisin in liquid culture. J. AOAC Int. 77:506-511.

Desjardins, A., and Hohn, T. 1997. Mycotoxins in plant pathogenesis. Mol. Plant-Microbe Interact. 10:147-152.

Desjardins, A., and Plattner, R. 2000. Fumonisin $\mathrm{B}_{1}$-nonproducing strains of Fusarium verticillioides cause maize (Zea mays) ear infection and ear rot. J. Agric. Food Chem. 48:5773-5780.

Desjardins, A., Plattner, R., Nelsen, T., and Leslie, J. 1995. Genetic analysis of fumonisin production and virulence of Gibberella fujikuroi mating population A (Fusarium moniliforme) on maize (Zea mays) seedlings. Appl. Environ. Microbiol. 61:79-86.

Desjardins, A., Plattner, R., and Proctor, R. 1996. Linkage among genes responsible for fumonisin biosynthesis in Gibberella fujikuroi mating population A. Appl. Environ. Microbiol. 62:2571-2576.

Gilchrist, D. 1998. Programmed cell death in plant disease: The purpose and promise of cellular suicide. Annu. Rev. Phytopathol. 36:393-414.

Harris, L., Desjardins, A., Plattner, R., Nicholson, P., Butler, G., Young, J., Weston, G., Proctor, R., and Hohn, T. 1999. Possible role of trichothecene mycotoxins in virulence of Fusarium graminearum on maize. Plant Dis. 83:954-960.

Jardine, D., and Leslie, J. 1999. Aggressiveness to mature maize plants of Fusarium strains differing in ability to produce fumonisin. Plant Dis. 83:690-693.

Lamprecht, S. C., Marasas, W. F. O., Alberts, J. F., Cawood, M. E., Gelderblom. W. C. A., Shephard, G. S., Thiel, P. G., and Calitz, F. J. 1994. Phytotoxicity of fumonisins and TA-toxin to corn and tomato. Phytopathology 84:383-391.

Marasas, W. 2001. Discovery and occurrence of the fumonisins: A historical perspective. Environ. Health Perspect. 109:239-243.

Munkvold, G., and Desjardins, A. 1997. Fumonisins in maize: Can we reduce their occurrence? Plant Dis. 81:556-565.

Munkvold, G. P., McGee, D. C., and Carlton, W. M. 1997. Importance of different pathways for maize kernel infection by Fusarium moniliforme. Phytopathology 87:209-217.

Nelson, P., Marasas, W., and Toussoun, T. 1983. Fusarium Species: An Illustrated Manual for Identification. The Pennsylvania State University Press, University Park, U.S.A.

Nelson, P., Plattner, R., Shackelford, D., and Desjardins, A. 1991. Production of fumonisins by Fusarium moniliforme strains from various substrates and geographic areas. Appl. Environ. Microbiol. 57:2410-2412.

Panaccione, D., Johnson, R., Wang, J., Young, C., Damrongkool, P., Scott, B., and Schardl, C. 2001. Elimination of ergovaline from a grass-Neotyphodium endophyte symbiosis by genetic modification of the endophyte. Proc. Natl. Acad. Sci. U.S.A. 98:12820-12825.

Proctor, R., Desjardins, A., Plattner, R., and Hohn, T. 1999. A polyketide synthase gene required for biosynthesis of fumonisin mycotoxins in Gibberella fujikuroi mating population A. Fungal Genet. Biol. 27:100-112.

Reid, L., Hamilton, R., and Mather, D. 1996. Screening maize for resistance to Gibberella ear rot. Pages 1-40 in: Technical Bulletin 1996-5E. Agriculture \& Agri-food Canada, Ottawa, Ontario, Canada.

Ritchie, S. W., Hanway, J. J., and Benson, G. O. 1997. How a corn plant develops. Iowa State Univ. Ext. Special Rep. No. 48.

Schardl, C. 1996. Epichloe species: Fungal symbionts of grasses. Annu. Rev. Phytopathol. 34:109-130.

Seo, J.-A., Proctor, R., and Plattner, R. 2001. Characterization of four clustered and coregulated genes associated with fumonisin biosynthesis in Fusarium verticillioides. Fungal Genet. Biol. 34:155-165.

Sydenham, E., Shephard, G., and Thiel, P. 1992. Liquid chromatographic determination of fumonisins $B_{1}, B_{2}$, and $B_{3}$ in foods and feeds. $J$. AOAC Int. 75:313-318. 